\title{
Transition of Attention in Terminal Area NextGen Operations Using Synthetic Vision Systems
}

\author{
Kyle K. E. Ellis ${ }^{1}$, Lynda J. Kramer ${ }^{1}$, Kevin J. Shelton ${ }^{1}$, J.J. (Trey) Arthur III ${ }^{1}$, \\ and Lance J. Prinzel III ${ }^{1}$ and Robert M. Norman ${ }^{2}$ \\ NASA Langley Research Center ${ }^{1}$ \\ Hampton, VA \\ Boeing Research and Technology ${ }^{2}$ \\ Seattle, WA
}

This experiment investigates the capability of Synthetic Vision Systems (SVS) to provide significant situation awareness in terminal area operations, specifically in low visibility conditions. The use of a Head-Up Display (HUD) and Head-Down Displays (HDD) with SVS is contrasted to baseline standard head down displays in terms of induced workload and pilot behavior in 1400 RVR visibility levels. Variances across performance and pilot behavior were reviewed for acceptability when using HUD or HDD with SVS under reduced minimums to acquire the necessary visual components to continue to land. The data suggest superior performance for HUD implementations. Improved attentional behavior is also suggested for HDD implementations of SVS for low-visibility approach and landing operations.

\section{INTRODUCTION}

NASA Langley Research Center (NASA LaRC) and the Department of Transportation/Federal Aviation Administration (DOT/FAA) are conducting collaborative research to ensure effective technology development and regulatory guidance for Synthetic Vision Systems/Enhanced Flight Vision Systems (SVS/EFVS) advanced cockpit vision technologies in Next Generation Air Transportation System (NextGen) operations. These technologies have the potential to provide an additional margin of safety and aircrew performance to enable operational improvements for low visibility surface, arrival, and departure operations in the terminal environment with equivalent efficiency as visual operations.

Synthetic Vision is the display of a "computer-generated image of the external scene topography from the perspective of the flight deck that is derived from aircraft attitude, highprecision navigation solution, and database of terrain, obstacles and relevant cultural features” (FAA 2011). This technology has been shown to increase the level of terrain and situation awareness (SA) by displaying intuitive visual information that mimics the out-the-window visuals when none is actually available due to poor visibility. The outcome is documented improvement of safety against controlledflight-into-terrain and possibly, loss-of-control accidents (Prinzel, et al., 2000; Bateman, 2010).

SVS technology exists today and is certified for general aviation aircraft and numerous business jets such as the Gulfstream V (Freeman 2002); however, despite significant improvements in terrain and situation awareness, at present, no additional 'operational credit' is provided from its installation or use.

\section{The Current Study}

A fixed-base simulation experiment was conducted to investigate if a lower decision height or reduced visibility minima is warranted by the use of SVS (Figure 1), thus providing 'operational credit'. The experiment was designed around the current operating regulations for landing under Instrument Flight Rules, as defined under 14 Code of Federal Regulations (CFR) §91.175 and following the precedent established under FAA Order 8400.13 ("Procedures for the Evaluation and Approval of Facilities for Special Authorization Category I Operations and All Category II and III Operations"). The premise was that integrated terrain and symbology presentation of the SVS merits operational credit since it:

1) Reduces flight technical error and promotes a stabilized approach; thus, ensuring that the aircraft will be in the proper position at the end of the instrument segment with minimal transition from which to complete the flare and landing;

2) Eases the pilot's transition from the instrument to the visual flight segment, by improving the pilot's ability to correctly recognize and identify the required visual references as per 14 CFR $\$ 91.175$ because the intuitive visual-like SVS information promotes efficient out-the-window visual search.

A key element of this work is quantifying the pilots' distribution of attention and their workload and ability to transition from the instrument segment to the visual segment during an instrument approach to landing in low visibility conditions. This work also addressed the influence of head-up display (HUD) or head-down display (HDD) implementations. 


\section{METHOD}

\section{Flight Test Equipment}

Simulation testing took place in NASA LaRC's Research Flight Deck (RFD), modeled after current state-of-the-art aircraft with four 10.5” by 13.25 ” liquid crystal color displays (Figure 2). The RFD is a full-mission simulator with hydraulic-actuated side-stick control inceptors. The aircraft model was a Boeing 757-200.

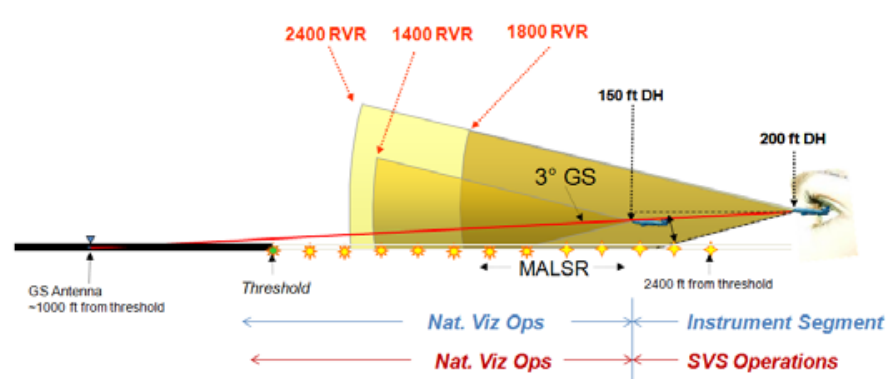

Figure 1. Proposed SVS Ops Change

The out-the-window (OTW) visual scene is generated by an Evans and Sutherland image generator and collimated projection system. The OTW field-of-view is $200 \mathrm{deg}$. horizontal (H) by $40 \mathrm{deg}$. vertical $(\mathrm{V})$ at 26 pixels per deg.

The four HDDs were populated with the Pilot Flying (PF), Primary Flight Display (PFD), the PF Navigation Display (ND), the Pilot Monitoring (PM) ND, and the PM PFD. The content on these displays was experimentally dependent, but followed standard PFD/ND design protocol.

The left (PF) seat of the RFD is equipped with a Flight Dynamics HGS-4000 HUD, collimated and subtending a $26^{\circ}$ $\mathrm{H}$ by $21^{\circ} \mathrm{V}$ field-of-view. The HUD projects imagery in stroke and raster format for symbology and SVS imagery, respectively. Pilots were able to "declutter" the stroke and raster imagery independently as desired using a four-button castle switch located on the sidestick inceptor.

A 4-camera Smart Eye ${ }^{\mathrm{TM}}$ head and eye tracking system was installed in the left seat of the RFD to capture quantitative data of PF head and eye movement.

\section{Simulator Database}

The test was simulated at Chicago O'Hare International Airport. Weather effects were experimentally varied, but for this analysis/paper, the weather and associated visibility, measured by Runway Visibility Range (RVR), were held constant. Calligraphic projection simulated a Medium intensity Approach Lighting System with runway alignment indicator. Runway centerline (CL) and touchdown zone (TDZ) lights were also experimentally varied.

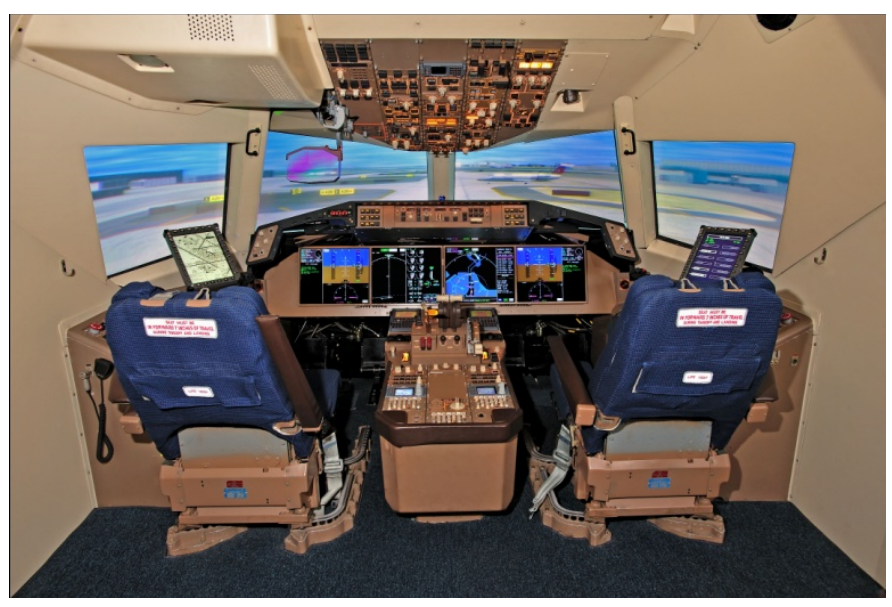

Figure 2. NASA Research Flight Deck

Altitude call-outs were given over the flight deck audio system, including "500 feet", "approaching minimums" and "minimums", referencing ownship to height above threshold (HATh). Additionally, call-outs of radar altitude starting at $100 \mathrm{ft}$ in $10 \mathrm{ft}$ increments to touchdown were also included.

\section{Experimental Design}

The experiment investigated, among other objectives, three comparisons of SVS application and their effect on pilot attention during transition to visual acquisition on short final: 1) SVS location (HUD vs. HDD), 2) SVS equipage (SVS HDD vs. No-SVS HDD, Figure 3), and 3) Effect of single versus dual crew operations. Comparative "baseline" conditions were those without SVS imagery. The experiment matrix is shown in Table 1 with the comparisons using the numbering above.

The single versus dual crew operations conditions were run to assess the PF's attentional, behavioral, and visual scanning with and without a PM assisting with verbal observations and call-outs, especially in the instrument-to-visual transition. The influence of SVS on this condition can only be inferred since baseline runs were not flown in single operations.

HUD presentation of SVS was shown in green raster on the combiner glass of the HUD, projected to be conformal to the environment from the pilot eye point (Figure 3) with overlaid symbology.

Variance in CL and TDZ lighting was included as an even distribution across conditions.

This research utilized 11 scenarios presented to each crew to address all required configuration comparisons, 8 crewed operations and three single pilot operations. A total of four HUD scenarios were presented in the crewed operation, with two baseline HUD and two SVS HUD scenarios (one each with the TDZ and CL lights varied). Four HDD scenarios were presented, two with baseline and two SVS configurations scenarios (one each with the TDZ and CL lights varied). 
Single pilot scenarios evaluated only the SVS configuration, totaling three scenarios.

Initial conditions for all scenarios positioned the aircraft lined up for the approach on final inside the final approach fix. Four wind profiles were generated to provide headwind, tailwind and crosswind elements no greater than $15 \mathrm{kts}$. All scenarios were presented with an OTW visibility of $1400 \mathrm{ft}$ and a decision height of 150 HATh.

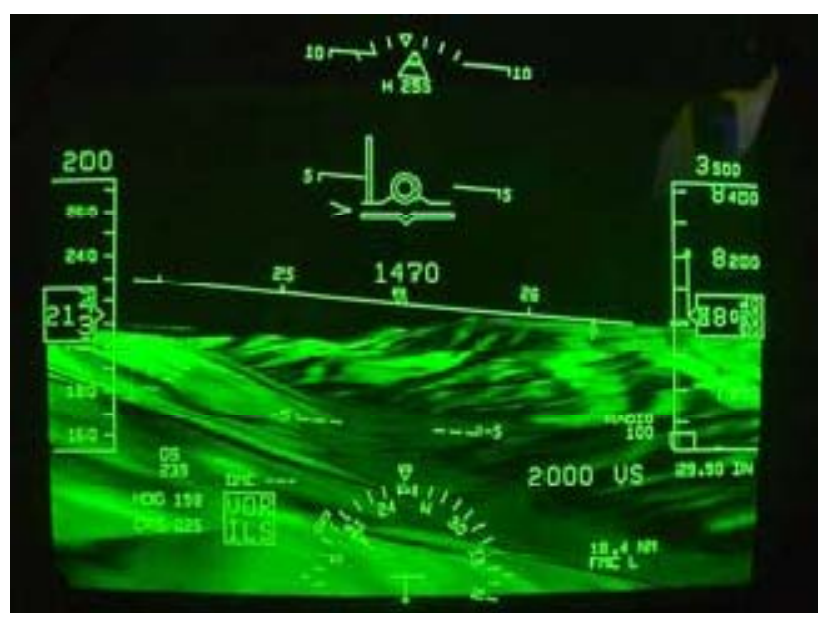

Figure 3. HUD with SVS and Symbology
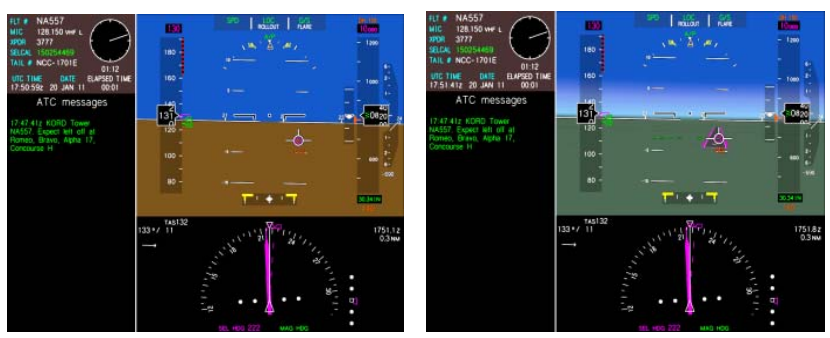

Figure 4. Baseline (left) and SVS (right) HDD Configurations

Table 1. Experiment Matrix

\begin{tabular}{|cc|c|c|}
\hline Display & $\begin{array}{c}\text { Vision } \\
\text { System }\end{array}$ & Crewed & Single \\
\hline \hline \multirow{2}{*}{ HUD } & None & 1 & \\
\cline { 2 - 4 } & SV & 1 & \\
\hline \multirow{2}{*}{ HDD } & None & 1,2 & \\
\cline { 2 - 4 } & SV & $1,2,3$ & 3 \\
\hline
\end{tabular}

\section{Evaluation Pilots}

24 pilots making up 12 crews were used as evaluation pilots. Each pilot held an Airline Transport Pilot rating and had at least 100 hours pilot-in-command HUD experience. Preference was given to pilots with operational experience with SVS and EVS. Pilots were paired by current employer to minimize crew coordination issues that might otherwise arise due to variance in Standard Operating Procedures and Crew Resource Management training. Pilots were trained in a preflight briefing and in the simulator for the various display configurations and crew procedures for SVS and EVS operations under the evaluation criteria.

\section{Results}

\section{Pilot Workload}

A standardized Air Force Flight Test Center workload scale questionnaire was administered after each scenario (Gawron, 2000).

In the first statistical comparison of SVS location (HUD versus $H D D)$, there was a significant difference $(F(1,68)=5.71$, $p=0.020$ ) indicating the utilization of a HUD effectively reduces the PF workload from 3.68 (HDD) to 3.13 (HUD).

The second statistical comparison evaluating SVS equipage indicated there was no significant effect on PF workload. SVS on a HDD yielded an average 3.64 relative to a baseline condition HDD (No SVS) average of 3.74.

The third comparison - effect of crewed versus single pilot operations - no statistically significant differences were found. Crewed operations yielded an average workload of 3.64 and single pilot operation yielded a slightly increased average workload of 3.78 .

\section{Evaluation Pilot Behavior}

Pilot attention and visual behavior was inferred by the head and eye tracking data to determine when pilots transitioned from instrumentation to OTW visual to acquire the runway environment to continue to landing. Metrics used to evaluate pilot behavior included; head-up percentage, transition count between OTW and head-down. For HDD concepts only, the transition glance - the altitude at which the PF first looked OTW - and the "full transition" OTW HATh altitude was also determined to evaluate variance in pilots' visual acquisition behavior.

Pilot eye and head tracking data was broken into five segments; three in the approach phase (Instrument, Instrument-to-visual, and Visual segments) and two in the landing phase (Flare and Landing, including roll-out, segments), to provide logical contrast across the phases of final approach and landing (Figure5).

\section{SVS Location Comparison (HUD vs. HDD)}

Eye/head tracking results showed significant effects on headup percentage across all three approach segments $(\mathrm{F}(11,1)=34.13, p<0.000)$, no effect between locations during the flare segment, and significance in the landing segment $(\mathrm{F}(11,1)=6.41, p=0.028)$. HDD concept findings indicate that pilots during the visual segment still remain head-down on 
average $30 \%$ of the time, scanning information available on the PFD. The landing segment saw statistical significance, however, operational significance is weak, with conditions varying between approximately 85 - 95\% head up.

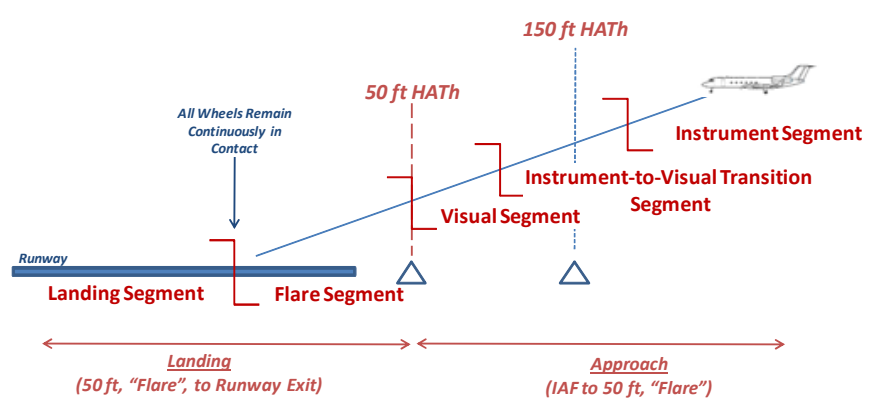

Figure 5. Eye Tracking Analysis Segments

Transition count analysis indicated similar significance findings to that of the head-up percentage, with the exception of the landing segment where no statistical significance existed between location conditions. However, the landing segment did show significance $(\mathrm{F}(11,1)=5.85, p=0.034)$ with the presence of TDZ/CL lights driving up the number of transitions made regardless of SVS location.

\section{SVS HUD vs. Baseline HUD Comparison}

Head-up percentage analysis results show no significant difference in pilot attentional behavior between SVS and Baseline display concepts presented on the HUD across all segments of approach and landing. Head-up percentage averages ranged between $87 \%$ and $98 \%$ for the Baseline HUD concept, and $91 \%$ and $99 \%$ for the SVS HUD concept.

Transition count analysis indicates no significant effect between SVS and Baseline display concepts presented on the HUD across all segments of approach and landing. Transition count averages ranged between 0.13 and 5.5 for the Baseline HUD concept, and 0.12 and 4.4 for the SVS HUD concept.

\section{SVS HDD vs. Baseline HDD Comparison}

Head-up percentage analysis results show no significant effects during any segment with the exception of the visual segment $(F=(11,1)=7.39, p=0.020)$, in which SVS drove pilot attention out-the-window $71 \%$ compared to the baseline average of $58 \%$ during this segment.

Transition count analysis indicates no significant effects between SVS HDD and Baseline HDD concepts across all of the flight segments. This data suggests the presence of SVS do not alter pilot OTW transition behavior relative to the baseline HDD concept. TDZ/CL lights did show a significant effect during the landing segment $(\mathrm{F}=(11,1)=78.23, p<0.000)$, again suggesting the presence of TDZ/CL lights drive up the number of OTW transitions regardless of HDD concept.

\section{SVS HDD Crewed vs. Single Comparison}

Head-up percentage analysis results show no significant effects during any segment with the exception of the landing segment $(F(11,1)=15.69, p=0.002)$, during which single crew operation drives pilot attention inside the cockpit $25 \%$ of the time relative to crewed operation where PF attention was inside the cockpit $12 \%$ of the time, on average.

Transition count analysis indicates similar results to that of the head-up analysis, showing only the landing segment to contain significant difference between crewed and single pilot operations $(\mathrm{F}(11,1)=20.42, p=0.001)$. Single pilot operation drives the pilot to transition between HDD and OTW on average approximately 9 times relative to the crewed operations average of 4.5 .

\section{SVS HDD vs. Baseline HDD Concept Transition HATh}

Pilot initial glance transition data showed statistical significance between the SVS HDD and Baseline HDD concepts $(\mathrm{F}(1,70)=6.98, p=0.010)$, indicating SVS concepts keep pilot attention inside the flight deck until closer to the DH. Baseline initial glance transition occurred at $377 \mathrm{ft}$ while SVS initial glance transitions occurred at $266 \mathrm{ft}$, on average.

The full transition analysis showed no significant difference between Baseline and SVS concepts, with values of $192 \mathrm{ft}$ and $164 \mathrm{ft}$, respectively. While not statistically significant, these values still show the same trend observed in the initial glance data, suggesting SVS maintains attention inside the flight deck until closer to the $\mathrm{DH}$.

There was no significant difference between crewed and single pilot operations in initial glance HATh with both occurring above the $\mathrm{DH}$ on average at $266 \mathrm{ft}$ and $270 \mathrm{ft}$, respectively. Full visual transition OTW was not statistically significant; however, data shows single pilot full transition does not occur until below the $\mathrm{DH}$ on average $(130 \mathrm{ft})$, while crewed operations fully transitioned OTW above the DH - at $164 \mathrm{ft}$ on average.

\section{Pilot Comments}

Pilot comments were collected during and after the experiment to collect professional insight into use of the systems and their impact on performance, workload, and acceptability/usability. Pilots ranked their low-visibility operations display concept preferences as follow: 1. SVS HUD, 2. Baseline HUD, 3. SVS HDD, 4. Baseline HDD. These results suggest the display location to be paramount in selection criteria, followed by display concept, with SVS being more preferable than baseline. These preferences were statistically significant across the display concepts $\left(\chi^{2}(4)=31.145, p<0.001\right)$.

\section{Discussion}

These analyses are intended to quantify the pilots' distribution of attention and their workload and ability to transition from the instrument segment to the visual segment during an instrument approach to landing and rollout in low visibility conditions. This work also addressed the influence of HUD or HDD implementations of SVS, and crewed or single pilot operations while utilizing SVS. Also considered is the 
influence of the runway environment (from the presence or absence of TDZ and CL lights).

The results of the analyses, as expected, provide a strong argument supporting the utilization of a HUD. It is not only preferred by pilots, but through quantitative comparison, it is effective in keeping pilot attention out-the-window and minimizing the time spent transitioning between instruments on the HDD and OTW. This behavior is particularly prominent in the instrument-to-visual and visual flight segments. The data indicates that, on average, the PF went head-down $30 \%$ of the time even in the visual segment. The benefit of HUD usage is further supported by a reduction in reported pilot workload.

No significant attentional variance was found when SVS was shown on the HUD. Future work will assess HUD clutter and masking effects. These influences are of critical importance in determining the safety impact of allowing pilots to utilize HUD-projected SVS. Reduced minima effectively reduce the time between decision height and touchdown, thus, increasing the criticality of acquiring the runway environment.

For HDD concepts, introducing SVS resulted in increasing the time spent OTW during the visual segment of flight and no significant difference in OTW-HDD transition counts when compared to the baseline. These results suggest the use of SVS provides an increased SA to pilots during the time critical short final in low visibility, allowing them to quickly glance at the HDD and obtain the necessary information to then again transition OTW to acquire the runway environment. SVS HDDs kept pilot attention inside the flight deck until much closer to the DH, possibly suggesting the SVS imagery affords the pilot a more decisive transition cue at the published $\mathrm{DH}$ when visual acquisition of the runway environment is increasingly likely in poor visibility conditions. There was no significant variance in workload reported between SVS and Baseline HDD conditions signifying pilots easily adapt to use of the SVS display concepts.

Comparing SVS concepts for crewed and single pilot operations, showed the influence of the PM's assistance. In the landing and roll-out, single pilot operations require the pilot to transition twice as frequently between OTW and HDD to achieve the proper SA to complete the task of exiting the runway at the proper turnoff. This is reflected in a $10 \%$ reduction of head up time when the pilot in command should maximize visual attention OTW to scan for surface traffic. The PF was drawn into the cockpit to reference a HDD-airport moving map for awareness of positioning on the runway and the location of the expected taxi exit. In crewed operations, the PM provided verbal assistance and direction. Transition HATh analysis shows that single pilot operations were slower in fully transitioning attention OTW compared to crewed operations. A full transition is indicative of the runway environment being visually acquired allowing pilots to complete the visual approach. These findings may suggest single pilots were more workload saturated attempting to monitor the approaching $\mathrm{DH}$ as well as the precision guidance than when flying as a crew. However, these quantitative data disassociate from the insignificant differences in subjective PF workload ratings between crewed and single pilot operations. This leads to the argument that while pilots may not feel a difference in perceived workload, operationally there is a significant impact. These results strongly suggest that the presence of a crew member significantly effects pilot transition behavior by reducing the number of times a pilot is driven to reference information inside the flight deck while maintaining proper safe attention out-the-window.

\section{Acknowledgements}

This work was conducted through joint sponsorship of NASA, under the Aviation Safety Program, Vehicle Systems Safety Technology project, and the FAA under the Human Factors Research and Development Project for NextGen. The support of Mr. Randall E. Bailey, Dr. Steven D. Young, NASA, Dr. Tom McCloy, Ms. Terry Stubblefield, and Mr. Dan Herschler, FAA, is gratefully appreciated.

\section{References}

Bailey, R. E., L. J. Kramer, and S. P. Williams. "Enhanced Vision for All-Weather Operations Under NextGen." SPIE Defense Security and Sensing Conference Proceedings. Orlando, FL: SPIE, 2010. vol. 7689.

Bateman, D. "Some Thoughts on Reducing the Risk of Aircraft Loss of Control," paper presented at the 2010 AIAA Guidance, Navigation, and Control Conference, AIAA Paper No. AIAA 2010-8139, Toronto, Canada, 2 - 5 August 2010.

FAA. Code of Federal Regulations. February 15, 2011.

FAA. Procedures for the Evaluation and Approval of Facilities for Special Authorization Category I Operations and All Category II and III Operations. October 22, 2009.

Freeman, G. "Gulfstream Enhanced Vision System." Symposium Proceedings - Society of Experimental Test Pilots. Society of Experimental Test Pilots, 2002. 154-168.

Gawron, V. J. "Human Performance Measures Handbook" Lawerence Erlbaum and Assoicates, New Jersey. 2000.

Hemm, R., G. Shapiro, D. Lee, J. Gribko, and B. Glaser. Beneift Estimates of Terminal Area Productivity Program Technologies. Final Report, Hampton, VA: NASA Langley Research Center, 1999.

Prinzel, L J., J.R. Comstock, D. Elliot, L.J. Kramer, L.B. Glaab, and R.V. Parrish. "The NASA Synthetic Vision Systems research project." Proceedings of the 4th Human Performance, Situation Awareness, and Automation: UserCentered Design for the New Millennium Conference Proceedings. Savannah, GA: North Carolina State University, SA Technologies, Mississippi State University, 2000. 359. 